\title{
Enhancing Cooperation in Distributed Information Systems using Conviviality and Multi-Context Systems
}

\author{
Patrice Caire $^{1}$ and Antonis Bikakis ${ }^{2}$ \\ ${ }^{1}$ University of Namur, PReCISE Research Center, Computer Science Department, Belgium \\ ${ }^{2}$ Department of Information Studies, University College London
}

\begin{abstract}
Modern information systems are characterized by the distribution of information and services among several autonomous heterogeneous entities. A major requirement for the success of such systems is that participating entities cooperate by sharing parts of their local knowledge. This paper presents a novel approach for modeling and enhancing cooperation in distributed information systems, which combines two formal models from the field of Knowledge Representation and Reasoning: a conviviality model and Multi-Context Systems. Our aim is two-fold. First, we develop a combined model for context-based representation and cooperation. Second, we provide the means for measuring cooperation leading to the design and evaluation of more convivial systems.
\end{abstract}

Key words: Distributed Artificial Intelligence, Knowledge Representation and Reasoning, Conviviality, Multi-Context Systems, Social Dependence Networks.

\section{Introduction}

Facilitating cooperation is a major challenge for distributed information systems. However, when cooperation is achieved among human and /or artificial entities, e.g. by enabling information exchange between them, such systems allow participating entities to enrich their local knowledge both in terms of quality and quantity. Moreover, for environments where the communication between entities cannot be guaranteed, e.g. Ambient Intelligence environments, it is also important for an entity to maintain multiple connections, so that it has more than one alternative choice to cooperate.

Systems that can be classified as distributed information systems, may include distributed databases, peer-to-peer systems, web social networks, mobile computing systems, the Semantic Web and many others. Despite their major differences with respect to their architectures, their aims and the nature of participating entities, all these systems are characterized by some common features, namely: (a) the available knowledge is distributed among several, possibly heterogeneous, human or artificial entities; (b) there is an available means of communication through which participating entities communicate and cooperate by sharing parts of their local knowledge; (c) each entity remains autonomous in the sense that it may take decisions independently, e.g. regarding which entities to communicate with, share knowledge or, more generally cooperate with, which parts of its knowledge should remain private, etc.; and (d) the system is open and dynamic in the sense that various entities may join or leave the system at 
random times and without prior notice. As a result, the structure of the system and the available system knowledge typically vary over time.

Taking into account the aim and special features of distributed information systems, a major requirement for the success of such systems is that participating entities cooperate by sharing local knowledge. We hereby address this issue and formulate it with the following research question:

How to enhance cooperative Distributed Information Systems (DIS)?

This breaks down into the following subquestions:

1. How to model local knowledge and information exchange in DIS?

2. How to model cooperation in DIS?

3. How to enhance cooperation in such systems?

To address these questions we combine two different formal models from the field of Knowledge Representation and Reasoning: a conviviality model [1,2] and MultiContext Systems [3, 4].

The concept of conviviality, issued from social science, has many ambiguous and non-technical definitions; it has been associated with concepts including empathy, trust, solidarity, and security [5]. Here we use Illich's definition of conviviality as "individual freedom realized in personal interdependence" [6]. In distributed information systems, individual freedom is linked with the choice to keep personal knowledge and beliefs at the local level, while interdependence is understood as reciprocity, i.e. cooperation. Participating entities depend on each other to achieve the enrichment of their local knowledge.

On the other hand, Multi-Context Systems (MCS) are logical formalizations of distributed context theories connected through a set of mapping rules, which enable information flow between contexts. A context can be thought of as a logical theory - a set of axioms and inference rules - that models local knowledge. In distributed information systems, a context may formally describe the local knowledge and beliefs of an entity, while mappings represent the information exchange between different entities.

In this work we introduce the groundings for convivial information exchanges among the entities of Multi-Context Systems. We believe that the work, although still in its infancy, is advanced enough to provide a solid ground for further research in fields such as artificial social systems, distributed artificial intelligence and DIS.

The layout of this paper is structured as follows. In section 2, we introduce a motivating example from the field of Social Networks. In section 3, we provide the necessary background information on Multi-Context Systems and tools for conviviality. In section 4 , we present our model using the running example. In section 5, we summarize and describe the next steps of this work.

\section{Motivating Scenario}

In [7], the authors used a scenario from Social Networks to illustrate a representation model for distributed peer theories and an algorithm for distributed query evaluation in Multi-Context Systems. In this paper, we extend the scenario to illustrate how to model and enhance convivial information systems. 


\subsection{A Use Case from Social Networks}

Consider the following use case from the domain of social networks. It involves a university social network, called "uni.scholar.space". People studying or working for the university can become members of the network and form groups through which they can discuss their common interests, arrange meetings, share information and other resources, such as electronic books, journals, papers or reports. We first present a simple use case of the system. Then, we extend this use case, and show how we can enhance cooperation between the system's users.

Simple Case. Alice, Bob, Charlie and Dan are members of uni.scholar.space. They have created a group, which they use to share information about articles that are relevant to their research interests. Alice, who is the head of the group, has configured a software agent to crawl the Web and seek articles that are relevant to the group's interests. For each article, the agent collects specific information, e.g. the name of the journal that the article was published in, and its keywords. If the article matches Alice's preferences, the agent recommends the article to Alice. The other members of the group (Bob, Charlie and Dan) use their own software agents, which recommend articles to their users based on Alice's recommendations, but also encode and share their personal research knowledge.

Consider that Alice's agent finds a new article on the Web, which has been recently published in the "Ambient Intelligence" journal (an imaginary journal) and has two keywords: "Multi-Agent Systems" and "Semantic Web". Alice is interested in articles that combine the fields of "Computer Applications", "Artificial Intelligence" and "Distributed Computing". Alice's agent cannot match the article with Alice's preferences, and therefore cannot determine how to recommend the article. The agent, then, attempts to contact the agents of the other members of the group so that they can collectively decide whether the article may be interesting for the group.

Bob is an expert in distributed computing. In this case, he knows that Multi-Agent Systems is a distributed computing model and shares this information with Alice. Charlie specializes in the field of Knowledge Representation and Reasoning (KRR), which is a subfield of Artificial Intelligence. His agent identifies Semantic Web as a subfield of Knowledge Representation and Reasoning, and shares this knowledge with Alice's agent. Dan's main research field is Mobile Applications, a specific type of computer applications. He is not aware, though, whether the paper title ("Ambient Intelligence") or any of the two keywords ("Multi-Agent Systems" and "Semantic Web") may be linked to this field. Based on the available information, Alice's agent cannot determine whether the paper matches Alice's preferences. Figure 1(a) illustrates this simple case. A circle represents an agent acting on behalf of one of the users involved in the scenario, i.e., "A" for Alice, "B" for Bob and so on. Arrows indicate information exchanges among the agents.

Extended Case. Consider now, that the system accesses the public profile of another registered user, Emma, who is an expert in "Pervasive Computing", a type of mobile applications. The system recommends Emma as a contact to Dan, based on their common 


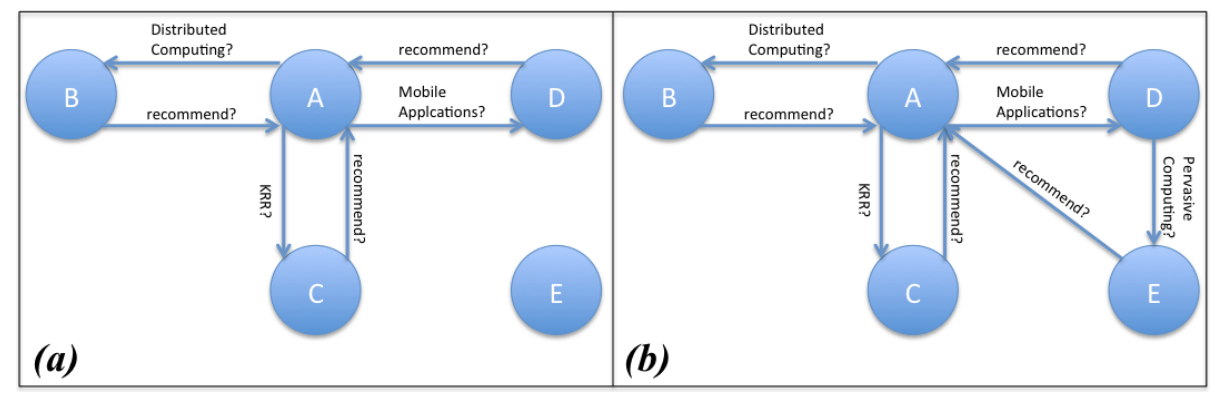

Fig. 1. Running example.

research interests. Emma knows that "Ambient Intelligence" is a journal specializing in pervasive computing, and shares this knowledge with Dan. Using this information, Alice's agent may now determine that the article should be recommended to Alice. It shares this recommendation with the agents of the other group members, and also with Emma's agent in counter-part for the information she provided to the group (Figure $1(b))$.

\subsection{Assumptions and requirements}

The implementation of the scenarios described above requires the combination of technologies from various fields including Software Engineering, Human-Computer Interaction, Computer Networks and Knowledge Representation and Reasoning (KRR). Our focus is on issues related to KRR. We implicitly make the follow simplifying assumptions for issues that are out of the scope of this paper.

- There is an available infrastructure for communication between the users' devices - the university network. The network is assumed to support various types of communications, both wired and wireless, and various types of devices, ranging from desktop computers to mobile phones.

- Each agent is aware of parts of the knowledge that the other agents possess. This feature is provided by uni.scholar.space to its registered members. Typically, as in all social networks today, each user has her own public profile, and may share more information with her contacts, or members of common groups.

- Each device has the required computing capabilities to conduct some simple reasoning tasks.

The specific problems that we try to address are:

- How to develop a common model not only for the representation of the agents' local knowledge, but also for the sharing of knowledge through information exchange.

- How to extend the system so that it can facilitate and enhance cooperation among its members, whereby providing them with more opportunities for information exchanges. 


\section{Background}

\subsection{Multi-Context Systems}

Multi-Context Systems (MCS [3,4]) are logical formalizations of distributed context theories connected through a set of mapping rules, which enable information flow between contexts. A context can be thought of as a logical theory - a set of axioms and inference rules - that models local knowledge. Together with the Propositional Logic of Context (PLC $[8,9])$ they are considered as the most prominent approaches in the field of contextual reasoning, which was introduced in AI by McCarthy [10] as an approach for the problem of generality. MCS have been argued to be most adequate with respect to the three dimensions of contextual reasoning (partiality, approximation, proximity) and shown to be technically more general than PLC [11].

Several distributed systems and applications have been developed on top of formal models of context, including (a) the CYC common sense knowledge base [12]; $(b)$ contextualized ontology languages, such as Distributed Description Logics [13] and COWL [14]; $(c)$ context-based agent architectures [15, 16]; and $(d)$ distributed reasoning algorithms for Mobile Social Networks [17] and Ambient Intelligence systems [18].

Recently, nonmonotonic extensions of MCS have been proposed to deal with context imperfections. Contextual Default Logic [19] extends MCS with default mapping rules to handle missing information and conflicts caused by mapping rules, while Contextual Defeasible Logic (CDL [20,21]) uses preferences to resolve potential inconsistencies caused by mapping rules. For the rest of the paper, we will use the representation model of CDL, though similar results may also be obtained for other MCS.

In CDL, the original Multi-Context Systems model is extended with defeasible rules, and a preference relation reflecting the trust each context assigns to other contexts. Specifically, CDL defines a MCS $C$ as a collection of distributed context theories $C_{i}$ : A context $C_{i}$ is defined as a tuple of the form $\left(V_{i}, R_{i}, T_{i}\right)$, where $V_{i}$ is the vocabulary used by $C_{i}$ (a finite set of positive and negative literals), $R_{i}$ is a set of rules, and $T_{i}$ is a preference ordering on $C$.

$R_{i}$ consists of two sets of rules: the set of local rules and the set of mapping rules. The body of a local rule is a conjunction of local literals (literals that are contained in $V_{i}$ ), while its head contains a local literal. There are two types of local rules: (a) Strict rules, of the form

$$
r_{i}^{l}: a_{i}^{1}, a_{i}^{2}, \ldots a_{i}^{n-1} \rightarrow a_{i}^{n}
$$

They express sound local knowledge and are interpreted in the classical sense: whenever the literals in the body of the rule $\left(a_{i}^{1}, a_{i}^{2}, \ldots a_{i}^{n-1}\right)$ are strict consequences of the local theory, then so is the conclusion of the rule $\left(a_{i}^{n}\right)$. Strict rules with empty body denote factual knowledge; (b) Defeasible rules, of the form

$$
r_{i}^{d}: b_{i}^{1}, b_{i}^{2}, \ldots b_{i}^{n-1} \Rightarrow b_{i}^{n}
$$

They are used to express uncertainty, in the sense that a defeasible rule $\left(r_{i}^{d}\right)$ cannot be applied to support its conclusion $\left(b_{i}^{n}\right)$ if there is adequate contrary evidence.

Mapping rules associate literals from the local vocabulary $V_{i}$ (local literals) with literals from the vocabularies of other contexts (foreign literals). The body of each such 
rule is a conjunction of local and foreign literals, while its head is labeled by a single local literal. A mapping rule is modeled as a defeasible rule of the form:

$$
r_{i}^{m}: a_{i}^{1}, a_{j}^{2}, \ldots a_{k}^{n-1} \Rightarrow a_{i}^{n}
$$

Finally, each context $C_{i}$ defines a partial preference ordering $T_{i}$ on $C$ to express its confidence in the knowledge it imports from other contexts. $T_{i}$ is modeled as a directed acyclic graph, in which vertices represent system contexts and arcs represent preference relations between the contexts that label the connected vertices. A context $C_{j}$ is preferred by $C_{i}$ to context $C_{k}$, denoted as $C_{j}>^{i} C_{k}$, if there is a path from vertex labeled by $C_{k}$ to vertex labeled by $C_{j}$ in $T_{i}$.

\subsection{Conviviality}

Computer systems have to be user friendly and convivial [22], a concept from the social sciences defined by Illich as "individual freedom realized in personal interdependence" [6]. Multiagent systems technology can be used to realize tools for conviviality when we interpret "freedom" as choice [2]. For example, if your research fields are rapidly evolving, and there is only one colleague who can provide you with updates, then you depend on this colleague for your updates, but if there several colleagues who can do so, then you do not depend on a single one. We say that there is more choice, and thus it is more convivial.

Tools for conviviality are concerned in particular with dynamic aspects of conviviality, such as the emergence of conviviality from the sharing of properties or behaviors whereby each member's perception is that their personal needs are taken care of [23, 24].

We measure conviviality by counting the possible ways agents have to cooperate, indicating degree of choice or freedom for these agents to engage in coalitions. Our coalitional theory is based on dependence networks [25,26], labeled directed graphs where the nodes are agents, and each labeled edge represents that the former agent depends on the latter one to achieve some goal. According to [1], conviviality may be measured by the number of reciprocity based coalitions that can be formed. Some coalitions, however, provide more opportunities for their participants to cooperate with each other than others, being thereby more convivial. To represent the interdependencies among agents in the coalitions, we use dependence networks.Abstracting from tasks and plans we define a dependence network as in definition $1[1,2]$ :

Definition 1 (Dependence networks). A dependence network is a tuple $\langle A, G$, dep, $\geq\rangle$ where: $A$ is a set of agents, $G$ is a set of goals, dep :A $\times A \rightarrow 2^{G}$ is a function that relates with each pair of agents, the sets of goals on which the first agent depends on the second, and $\geq: A \rightarrow 2^{G} \times 2^{G}$ is for each agent a total pre-order on sets of goals occurring in his dependencies: $G_{1}>_{(a)} G_{2}$.

We now recall the assumptions and requirements for conviviality measures introduced in [2]. Our assumptions are first, that cycles identified in a dependence network are considered as coalitions, and second, that the conviviality of a dependence network is evaluated in a bounded domain. When referring to cycles, we are implicitly signifying 
simple cycles (as defined in [27]), also discarding self-loops. Moreover, when referring to conviviality, we always refer to potential interaction not actual interaction.

Our requirements are first, that larger coalitions are more convivial than smaller ones, and second, that the more coalitions in the dependence network, the higher the conviviality measure, ceteris paribus.

Conviviality classification We further recall from [1,2] the conviviality classification proposed to allow an intuitive grasp of conviviality measures through a ranking of the dependence networks. Following are the five definitions of conviviality classes, from the absolute best to the absolute worst convivial networks. Figure 2, illustrates the different types of dependence networks that correspond to each conviviality class. The arrow on the top of the figure depicts the direction of increasing conviviality. The scale goes from the worst case (no conviviality) to the best case (maximal conviviality).

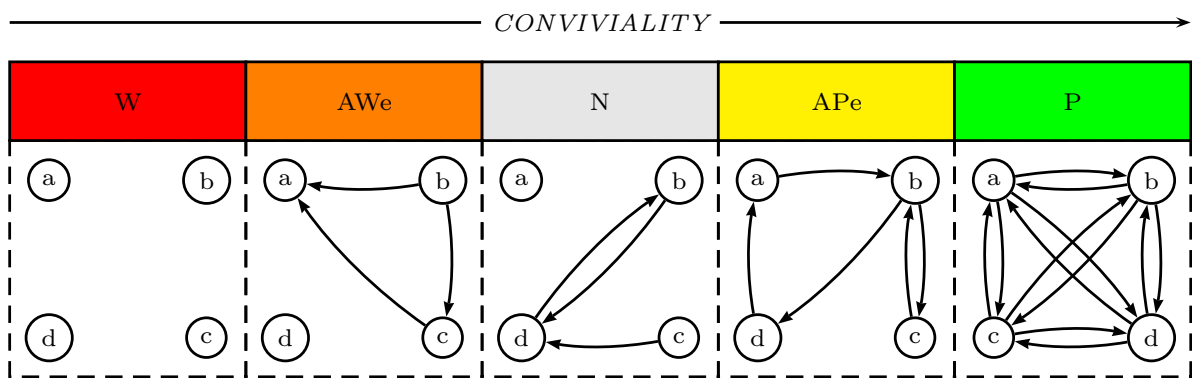

Fig. 2. Conviviality classes.

Definition 2 (P). A dependence network $D N$ is $P$ convivial (most convivial), iff all agents in $D N$ belong to all cycles, i.e., $\forall a_{i} \in A$ and $\forall c_{k} \in C, a_{i}$ is s.t. $a_{i} \in c_{k}$, where $C=\left\{c_{1}, \ldots, c_{l}\right\}$ is the set of all cycles.

Definition 3 (APE). A dependence network $D N$ is APE convivial, iff all agents in $D N$ belong to at least one cycle, i.e., $\forall a_{i} \in A, \exists c_{k} \notin C$, s.t. $a_{i} \in c_{k}$, where $C=$ $\left\{c_{1}, \ldots, c_{l}\right\}$ is the set of all cycles.

Definition 4 (N). A dependence network $D N$ is $N$ convivial, iff there exists at least one cycle in $D N$, and there is at least one agent not in a cycle, i.e., $\exists a, b \in A$ s.t. $a, b \in c_{k}$, where $c_{k} \notin C$, and $\exists d \in A$ s.t. $\quad d \notin c_{i}, \forall c_{i} \in C$, where $C=\left\{c_{1}, \ldots, c_{l}\right\}$ is the set of all cycles.

Definition 5 (AWE). A dependence network $D N$ is $A W E$ convivial, iff there is no cycle in $D N$, i.e., $C=\{\emptyset\}$, and s.t. $\exists \operatorname{dep}(a, b)=\left\{g_{i}\right\}$, where $a, b \in A$ and $g_{i} \in G$.

Definition 6 (W). A dependence network $D N$ is $W$ convivial (worst convivial), iff there is no dependency between the agents in $D N$, i.e., $\nexists \operatorname{dep}(a, b)=\left\{g_{i}\right\}$, where $a, b \in A$ and $g_{i} \in G$. 


\section{Model}

In this section, we first apply our Multi-Context System formalism to our motivating example. Then, building on our model, we apply our conviviality formalism. Finally, we discuss the resulting model.

\subsection{Formalization of the use case scenario as a Multi-Context System}

The use case scenario described in Section 2 can be formalized as follows using the language of Contextual Defeasible Logic. The group of agents can be defined as a MultiContext System consisting of five context theories. Each context theory encodes the local knowledge of an agent in terms of local rules, and the association between the local knowledge and the knowledge of the other agents in terms of mapping rules.

Simple Case. All agents share some common local knowledge, which refers to the information about the journal article:

$$
\begin{aligned}
& \rightarrow \text { ambient_intelligence } \\
& \rightarrow \text { semantic_web } \\
& \rightarrow \text { mas }
\end{aligned}
$$

Alice's local knowledge also includes her preferences, based on which the agent decides whether an article should be recommended:

$r_{A 1}^{d}:$ computer_applications $_{A}$, artificial_intelligence $_{A}$, distributed_computing $_{A} \Rightarrow$ recommend $_{A}$

Alice's agent also uses the following mapping rules to import information from the other agents:

$$
\begin{aligned}
& r_{A 2}^{m}: \text { distributed_computing } g_{B} \Rightarrow \text { distributed_computing } \\
& r_{A 3}^{m}: k r r_{C} \Rightarrow \text { artificial_intelligence } \\
& r_{A 4}^{m}: \text { mobile_applications } s_{D} \Rightarrow \text { computer_applications }_{A}
\end{aligned}
$$

Bob's agent uses one local strict rule to encode Bob's knowledge that "Multi-Agent Systems" is a distributed computing model $\left(r_{B 1}^{l}\right)$. It also uses a mapping rule $\left(r_{B 2}^{m}\right)$ to import the recommendation of an article from Alice's agent:

$r_{B 1}^{l}:$ mas $\rightarrow$ distributed_computing $g_{B}$

$r_{B 2}^{m}:$ recommend $_{A} \Rightarrow$ recommend $_{B}$

Charlie's knowledge that "Semantic Web" is a subfield of "Knowledge Representation and Reasoning" is encoded as a local strict rule $\left(r_{C 1}^{l}\right)$, while his agent also uses a similar mapping rule to import recommendations from Alice's agent $\left(r_{C 2}^{m}\right)$.

$r_{C 1}^{l}:$ semantic_web $\rightarrow k r r_{C}$

$r_{B 2}^{m}:$ recommend $_{A} \Rightarrow$ recommend $_{C}$ 
Dan's agent uses one mapping rule that imports recommendations for articles from Alice's agent $\left(r_{D 2}^{m}\right)$.

$r_{D 1}^{m}:$ recommend $_{A} \Rightarrow$ recommend $_{D}$

Extended Case. In the extended case, Dan's agent uses another one mapping rule $\left(r_{D 2}^{m}\right)$, which is suggested by the system and associates "pervasive computing" used by Emma with "mobile computing":

$r_{D 2}^{m}:$ pervasive_computing ${ }_{E} \Rightarrow$ mobile_computing ${ }_{D}$

Emma's agent uses a local strict rule that associates the "Ambient Intelligence" journal with "pervasive computing" $\left(r_{E 1}^{l}\right)$, and a mapping rule that imports recommendations for articles from Alice's agent $\left(r_{E 2}^{m}\right)$.

$r_{E 1}^{m}:$ ambient_intelligence $\rightarrow$ pervasive_computing ${ }_{E}$

$r_{E 2}^{m}:$ recommend $_{A} \Rightarrow$ recommend $_{E}$

\subsection{Conviviality in Multi-Context Systems}

In this subsection, we build on the Multi-context model proposed in subsection 4.1, and apply our conviviality formalism to the motivating example.

Simple case. Let $D N_{1}$, visualized in Figure 3 (a) be a dependence network corresponding to the coalition $C_{1}$, where: $C_{1}:\left\{\left(A, g_{1}, B, g_{2}\right),\left(A, g_{1}, C, g_{2}\right),\left(A, g_{1}, D, g_{2}\right)\right\}$, and where:

- Agents $A g=\{A, B, C, D, E\}$, respectively represent Alice, Bob, Charlie, Dan and Emma;

- Goals $G=\left\{g_{1}, g_{2}\right\}$, where the two main goals are the following: $\left\{g_{1}\right\}$ is to find the relevance of articles, and $\left\{g_{2}\right\}$ is to get recommended articles. (For easier reading, goals have been simplified to the two generic goals $g_{1}$ and $g_{2}$.)

- Dependencies are built from the Multi-context System rules, that is: $\operatorname{dep}(A, B)=\left\{g_{1}\right\}, \operatorname{dep}(A, C)=\left\{g_{1}\right\}, \operatorname{dep}(A, D)=\left\{g_{1}\right\}$ : agent A depends on agents $\mathrm{B}, \mathrm{C}$ and $\mathrm{D}$ to achieve its goal $\mathrm{g} 1$ : to find the relevance of articles; and $\operatorname{dep}(B, A)=\left\{g_{2}\right\}, \operatorname{dep}(C, A)=\left\{g_{2}\right\}, \operatorname{dep}(D, A)=\left\{g_{2}\right\}:$ agent $\mathrm{B}, \mathrm{C}$ and $\mathrm{D}$ depend on agent A to achieve their goals g2: to get recommended articles.

- Agents' preferences are inferred from the agents local knowledge as indicated in the Multi-Context System model.

Extended case Now, let $D N_{2}$, visualized in Figure 3 (b), be the dependence network corresponding to the coalition $C_{2}$, where:

$C_{2}:\left\{\left(A, g_{1}, B, g_{2}\right),\left(A, g_{1}, C, g_{2}\right),\left(A, g_{1}, D, g_{2}\right),\left(A, g_{1}, D, g_{1}, E, g_{2}\right)\right\}$, and where: 
- Agents $A g=\{A, B, C, D, E\}$ are as previously;

- Goals $G=\left\{g_{1}, g_{2}\right\}$, where the two main goals are the following: $\left\{g_{1}\right\}$ is to find the relevance of articles, and $\left\{g_{2}\right\}$ is to get recommended articles, as previously;

- Dependencies are built from the Multi-context System rules, that is: $\operatorname{dep}(A, B)=\left\{g_{1}\right\}, \operatorname{dep}(A, C)=\left\{g_{1}\right\}, \operatorname{dep}(A, D)=\left\{g_{1}\right\}$ : agent A depends on agents $\mathrm{B}, \mathrm{C}$ and $\mathrm{D}$ to achieve its goal $\mathrm{g} 1$ : to find the relevance of articles, but so does also agent $\mathrm{D}$ from $\mathrm{E}$ as $\operatorname{dep}(D, E)=\left\{g_{1}\right\}$;

$\operatorname{dep}(B, A)=\left\{g_{2}\right\}, \operatorname{dep}(C, A)=\left\{g_{2}\right\}, \operatorname{dep}(D, A)=\left\{g_{2}\right\}, \operatorname{dep}(E, A)=\left\{g_{2}\right\}:$ agent $\mathrm{B}, \mathrm{C}, \mathrm{D}$ and $\mathrm{E}$ depend on agent $\mathrm{A}$ to achieve their goals g2: to get recommended articles;

- Agents' preferences are inferred from the agents local knowledge as indicated in the Multi-Context System model.

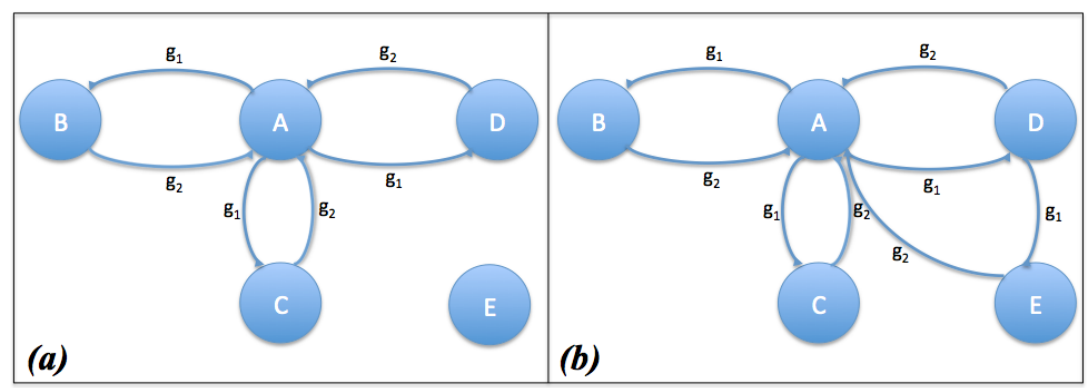

Fig. 3. Dependence networks $D N_{1}$ and $D N_{2}$.

\subsection{Discussion}

Consider the two dependence networks $D N_{1}$ and $D N_{2}$,respectively corresponding to the agents coalitions $C_{1}$ and $C_{2}$, illustrated in Figure 3. In $C_{1}$, some agents are in a cycle while one, namely $E$, is isolated and does not depend on any other agents. Hence, from Definition 4, $C_{1}$ belongs to the $N$ conviviality class. In contrast, all agents in coalition $C_{2}$ belong to at least one cycle. Hence, from Definition 3, $C_{2}$ belongs to the $A P e$ conviviality class. It is said to be Almost Perfectly convivial. All agents are engaged in reciprocal dependence relations: each one gives to the coalition and receives from it. All agents are pursuing goals and cooperate with at least one other agent to achieve the recommendations.

By recommending Emma to Dan, the system facilitates the information exchange among the agents and enhances the conviviality, making the system more effective and enjoyable to use. Moreover, by providing more choices for the agents to choose from, 
the system allows more possibilities for agents to reach their goals of gathering relevant information for their group members. We note that in the simple case, agent $A$ is a central point and there a bottleneck and a weakness for $C_{1}$, as if it leaves the coalition all cycles disappear. In the extended case, however, the choice, provided by involvement of agent $E$, contributes to more robust system.

\section{Summary}

In this paper we proposed a model for knowledge representation, information exchange and cooperation in distributed information systems. Using a use case scenario from the field of Social Networks we highlighted how cooperation can be enhanced in such systems using two formal models: a conviviality model and a context representation model called Multi-Context Systems. The proposed approach may be applied to various types of distributed information systems, such as mobile computing systems, Ambient Intelligence systems, social networks and the Semantic Web.

There are many different directions towards which this work may be extended. As we already argued in Section 3, the conviviality model may also be combined with other Multi-Context Systems, such as Contextual Default Logic [19] and managed MultiContext Systems [28]. The latter approach extends the definition of mappings to support more types of operations on contexts, such as revision and updates. Moreover, it is more general than CDL, since it allows contexts to use different representation models (e.g. relational databases, ontologies and logic programs).

Another interesting direction is to extend the proposed model with temporal aspects so that we are able to handle the dynamics of distributed information systems. To this direction, we will study the adoption of temporal dependence networks and temporal reciprocity measures introduced in [2].

Finally, considering the privacy requirements of users of Social Networks, Ambient Intelligence systems and other types of distributed information systems, we plan to study the tradeoff between privacy and conviviality in such systems. Both privacy protection and cooperation are essential for the adoption and success of such systems, therefore a tradeoff analysis will contribute to finding the right balance between the two properties in future information systems.

\section{References}

1. Caire, P.: New Tools for Conviviality: Masks, Norms, Ontology, Requirements and Measures. PhD thesis, Luxembourg University, Luxembourg (2010)

2. Caire, P., Alcade, B., van der Torre, L., Sombattheera, C.: Conviviality measures. In: 10th International Joint Conference on Autonomous Agents and Multiagent Systems (AAMAS 2011), Taipei, Taiwan, May 2-6, 2011. (2011)

3. Giunchiglia, F., Serafini, L.: Multilanguage hierarchical logics, or: how we can do without modal logics. Artificial Intelligence 65(1) (1994)

4. Ghidini, C., Giunchiglia, F.: Local Models Semantics, or contextual reasoning=locality+compatibility. Artificial Intelligence 127(2) (2001) 221-259

5. Caire, P.: How to import the concept of conviviality to web communities. International Journal of Web Based Communities (IJWBC) 6(1) (2009) 99-113 
6. Illich, I.: Tools for Conviviality. Marion Boyars Publishers, London (1974)

7. Bikakis, A., Antoniou, G.: Local and Distributed Defeasible Reasoning in Multi-Context Systems. In: RuleML. Volume 5321 of Lecture Notes in Computer Science., Springer (2008) 135-149

8. Buvac, S., Mason, I.A.: Propositional Logic of Context. In: AAAI. (1993) 412-419

9. McCarthy, J., Buvač, S.: Formalizing Context (Expanded Notes). In Aliseda, A., van Glabbeek, R., Westerståhl, D., eds.: Computing Natural Language. CSLI Publications, Stanford, California (1998) 13-50

10. McCarthy, J.: Generality in Artificial Intelligence. Communications of the ACM 30(12) (1987) 1030-1035

11. Serafini, L., Bouquet, P.: Comparing formal theories of context in AI. Artificial Intelligence 155(1-2) (2004) 41-67

12. Lenat, D.B., Guha, R.V.: Building Large Knowledge-Based Systems; Representation and Inference in the Cyc Project. Addison-Wesley Longman Publishing Co., Inc., Boston, MA, USA (1989)

13. Borgida, A., Serafini, L.: Distributed Description Logics: Assimilating Information from Peer Sources. Journal of Data Semantics 1 (2003) 153-184

14. Bouquet, P., Giunchiglia, F., van Harmelen, F., Serafini, L., Stuckenschmidt, H.: C-OWL: Contextualizing Ontologies. In: International Semantic Web Conference. (2003) 164-179

15. Parsons, S., Sierra, C., Jennings, N.R.: Agents that reason and negotiate by arguing. Journal of Logic and Computation 8(3) (1998) 261-292

16. Sabater, J., Sierra, C., Parsons, S., Jennings, N.R.: Engineering Executable Agents using Multi-context Systems. Journal of Logic and Computation 12(3) (2002) 413-442

17. Antoniou, G., Papatheodorou, C., Bikakis, A.: Reasoning about Context in Ambient Intelligence Environments: A Report from the Field. In: KR, AAAI Press (2010) 557-559

18. Bikakis, A., Antoniou, G., Hassapis, P.: Strategies for contextual reasoning with conflicts in Ambient Intelligence. Knowledge and Information Systems 27(1) (2011) 45-84

19. Brewka, G., Roelofsen, F., Serafini, L.: Contextual Default Reasoning. In: IJCAI. (2007) 268-273

20. Bikakis, A., Antoniou, G.: Defeasible Contextual Reasoning with Arguments in Ambient Intelligence. IEEE Trans. on Knowledge and Data Engineering 22(11) (2010) 1492-1506

21. Bikakis, A., Antoniou, G.: Partial Preferences and Ambiguity Resolution in Contextual Defeasible Logic. In: LPNMR. Volume 6645 of Lecture Notes in Computer Science., Springer (2011) 193-198

22. Caire, P., Villata, S., Boella, G., van der Torre, L.: Conviviality masks in multiagent systems. In: 7th International Joint Conference on Autonomous Agents and Multiagent Systems (AAMAS 2008), Estoril, Portugal, May 12-16, 2008, Volume 3. (2008) 1265-1268

23. Caire, P., van der Torre, L.: Convivial ambient technologies: Requirements, ontology and design. The Computer Journal 3 (2009)

24. Caire, P.: Designing convivial digital cities: a social intelligence design approach. AI and Society 24(1) (2009) 97-114

25. Castelfranchi, C.: The micro-macro constitution of power. Protosociology 18 (2003) 208269

26. Sichman, J.S., Conte, R.: Multi-agent dependence by dependence graphs. In: Procs. of The First International Joint Conference on Autonomous Agents \& Multiagent Systems, AAMAS 2002, ACM (2002) 483-490

27. Cormen, T.H., Leiserson, C.E., Rivest, R.L., Stein, C.: Introduction to Algorithms. 2nd edn. The MIT Press (2001)

28. Brewka, G., Eiter, T., Fink, M., Weinziel, A.: Managed Multi-Context Systems. In: IJCAI. (2011) 